\title{
Dicing with reality
}

\author{
Leslie Orgel
}

THE authors of this ambitious book, a translation from the German original Das Spiel, first published in 1975, have tried to make accessible to the general reader a group of related ideas which elsewhere are almost always treated with considerable mathematical sophistication. They touch on a very wide range of topics, but the core of the book is concerned with the development of the size and composition of populations in time. However, this is not a book about population genetics in any conventional sense; the populations may be comprised of anything from neutrons to humans. Once the laws or rules of production (birth), transition (mutation) and decay (death) are given, the "game" is defined - the size or shape of the pieces does not affect the outcome.

The major novelty of the treatment is the replacement of probability theory, with its theorems and derivations, by board games that exemplify the most important general principles. The idea as such is not new, but the consistent application of this idea and the complete absence of formal mathematical arguments make this a unique book. I found it a lot of fun to read and expect that many others will feel the same way.

But does the approach provide new insights or make old ones more intelligible? I think that the book will prove very useful for that large section of the scientific community which tends to quit when the mathematical going gets rough. Of course, if one tries to cover such a wide area in a book of modest length most of the detail must be excluded. In this context, board games are ideal, since the rules can rarely be detailed enough to model interesting situations in depth, but can often make transparent important general principles, for example those controlling the stability or instability of population size and composition.

An example may help to show how a board game can illustrate a general result. Suppose we have a square board, say a chess board, and cover it at random with 32 black and 32 white pieces. Then we roll a pair of eight-faced dice to choose a square at random. In variant 1 of the game, the piece on the chosen square is removed and replaced by a piece of the opposite colour. In variant 2 , a piece of the opposite colour (if one is present) is removed from any position on the board other than the chosen square and replaced by one of the same colour as that on the chosen square. Variant 1 is stable in the long term, in the
Laws of the Game: How the Principles of Nature Govern Chance. By Manfred Eigen and Ruthild Winkler. Translated by Robert and Rita Kimber. Pp.350. US ISBN 0-394-41806-9; UK ISBN 0-7139-1484-X. (Knopf/Allen Lane: 1982.)\$19.95, £14.95.

sense that even after a very long time a roughly equal number of black and white pieces will almost surely be left on the board. In the case of variant 2 , we may confidently expect that sooner or later all the squares will be occupied by pieces of the same colour, but we cannot predict which colour. This is a very familiar result, but it is instructive to watch the development of the distribution of pieces on the board for a few "games".

1 suspect that the mathematically inclined will still prefer the standard texts on population genetics, branching chain reactions, games theory and so on. For others, the present approach may provide a useful view of the wood, before they plunge into the trees.

While the core of this book is concerned with populations and growth, the authors make brief forays into every corner of the scientific and general cultural scene. As might be expected, some of these appeal to the present reviewer more than others. I thought the section on entropy outstandingly good, and many of the discussions of molecular evolution illuminating, but I couldn't make out a strong logical connection between the main content of the book and some of the later sections, for example, the avuncular advice offered in the section entitled "Limits".

Many will profit from this lively book. However, a little parental guidance on the relation between simple theories and complex reality may be advisable for novices to the game. One final thought - many of those who read this book will have access to a small computer. Advice on setting up these games for a computer, and perhaps some additional games especially designed with a computer in mind, would be a welcome addition to a second edition.

Leslie Orgel is a Senior Fellow at the Salk Institute, San Diego, California.

\section{Biogeography and natural experiments}

\section{D. Pigott}

The Changing Climate: Responses of the Natural Fauna and Flora. By Michael J. Ford. Pp.190. ISBN 0-04-574017-8. (George Allen \& Unwin: 1982.) £13.95, $\$ 27.50$.

ONE of the generally accepted principles of biogeography is that geographical distributions of plants and animals are primarily controlled by climate. It is therefore a fundamental weakness of the subject that so important a premise cannot readily be verified by experiment. Climate cannot easily be changed and the component factors of radiation, temperature, humidity and rainfall are physically interrelated so that their separate influences are only identified with great difficulty. Neither can whole ecosystems be moved from one climatic region to another, and experiments which consist of transferring individual organisms are so far removed from reality that the results must be treated with great caution.

It is in this context that climatic change assumes particular interest because it provides what are essentially natural experiments. As with all experiments both the change imposed and the response of the organisms must be accurately measured. The value of Michael Ford's book is that it brings together for the first time a large number of scattered observations on the response of organisms to natural variations in climate, and that it also provides an excellent summary of the climatic changes which have occurred during the period for which meteorological measurements are available (Chapter 3 ). In the following chapter evidence for changes before this period is also presented, but it is important to recognize that this largely, though not entirely, consists of fossil evidence for change in the distribution of organisms. There is, therefore, an inevitable circularity of argument.

The author avoids this danger by restricting his discussion in the rest of the book to responses of plants and animals to climatic changes since about 1900 . Although long-term variations during this period are small - for example a rise of mean annual temperature in the Northern Hemisphere of about $0.5^{\circ} \mathrm{C}$ from 1890 to 